\title{
APLICACIÓN EN COBERTERA DE LA MEZCLA FERTILIZANTE EN SUELOS CON DIFERENTES CONTENIDOS DE P OLSEN EN TRIGO (TRITICUM AESTIVUM L.)
}

\author{
APPLICATION OF A MIXTURE OF FERTILIZERS ON SOILS \\ WITH DIFFERENT CONTENTS OF P OLSEN IN CORN \\ (TRITICUM AESTIVUM $L$.)
}

\author{
Hernán Pinilla $Q .{ }^{1}$; Helga Herrera $P^{2}{ }^{2}$ Luis Herrera $F^{3}{ }^{3}$; Héctor Sanhueza $R^{4}$
}

\begin{abstract}
RESUMEN
Se evaluó el efecto de aplicaciones de mezcla de siembra al surco y al voleo incorporada en suelos con diferente disponibilidad de $\mathrm{P}$ Olsen en el rendimiento del trigo y en el contenido de $\mathrm{P}$ del suelo. No se produjeron diferencias significativas en rendimiento de trigo por efecto de la diversa forma de aplicación de la mezcla fertilizante. Las distintas dosis de $\mathrm{P}$ contenidas en las mezclas aplicadas en las temporadas 2005 y 2006 no registraron diferencias de rendimiento; sin embargo, en 2007 sí se observó respuesta. El nivel de P Olsen, en 2006 y 2007, se incrementó a medida que la dosis de P aplicada fue más alta, mientras que en 2005 no se produjo variación en el contenido de P Olsen

Palabras clave: Triticum aestivum, fósforo, forma aplicación, cobertera, surco, voleo.
\end{abstract}

\section{ABSTRACT}

The effect of $P$ applications to furrow and broadcast incorporated in soils with different $P$ Olsen availability was evaluated in the wheat yield and the soil $P$ content. There were no significant differences in wheat yield, by effect of different ways of applying the fertilizer mixture. Different doses of P applied in the seasons 2005 and 2006 showed no differences in performance, however, in 2007 it was observed response. The level of P Olsen was increased as the dose applied P was higher in 2006 and 2007, while in 2005 there was no response.

Key words: Triticum aestivum, wheat, phosphorus, furrow, broadcast incorporated, banding.

\section{INTRODUCCIÓN}

El contenido de fósforo disponible de los suelos volcánicos del sur de Chile suele ser limitante para la producción de trigo, por lo cual es común la aplicación de altas dosis de fertilizantes fosfatados en el surco de siembra. Este nutriente es el de más alta concentración en la mezcla de siembra, y va acompañado de cantidades variables de $\mathrm{N}, \mathrm{K}, \mathrm{Mg}$ $\mathrm{u}$ otros elementos de mayor movilidad que el $\mathrm{P}$ y que tienen la posibilidad de ser complementados por aplicaciones en cobertera. Por tanto, la aplicación de la mezcla de siembra en un surco al lado de la semilla se debe a la baja movilidad del fósforo, especialmente en suelos volcánicos (Rodríguez, 2001).

La eficiencia de uso del $\mathrm{P}$ varía con la forma de aplicación. En este sentido, las mayores respuestas en trigo se han obtenido fertilizando al surco de siembra (Berardo, 1999.) Sin embargo, con contenidos

1 Ingeniero Agrónomo, Magíster en Fertilidad de Suelos, Académico Investigador FCAF. Universidad de La Frontera. Chile. hpin@ufro.cl

2 Ingeniero Agrónomo. helga.herrera@sqm.com

3 Ingeniero Agrónomo, Magíster en Gestión y Manejo Productivo de Cereales. herreral@ufro.cl

4 Ingeniero Ejecución Agrícola. Académico FCAF, Universidad de La Frontera. Chile. hsanhue@ufro.cl 
moderadamente altos de $\mathrm{P}$, las aplicaciones al voleo e incorporadas podrían resultar de igual o mayor eficiencia (Mallarino, 1998). Esta forma de aplicación permite separar la localización de la semilla con la del fertilizante, lo que haría más eficiente el proceso de siembra.

En atención a la baja movilidad del $\mathrm{P}$ en el suelo, las aplicaciones localizadas tendrían mayor eficiencia que aplicaciones en superficie. Mallarino (1997) y Fariña (1997) señalaron que la respuesta a la fertilización al surco es superior a la aplicación al voleo cuando los suelos son muy deficientes en $\mathrm{P}$ o presentan alta capacidad de fijación. Sin embargo, cuando la fertilización al voleo se incorporó al momento de la siembra, ésta fue tan eficiente como la fertilización localizada (Mallarino y Borges, 2006). También se observó que a medida que el nivel de $\mathrm{P}$ del suelo aumenta, las diferencias entre aplicarlo al voleo o al surco disminuyen o no existen (Darwich, 1998).

Se debe tener presente que no existe una respuesta generalizada y única en relación a cuál es la forma más adecuada de aplicar el fertilizante fosforado, ya que la efectividad relativa de las aplicaciones en surco o al voleo varía dependiendo de situaciones específicas, como la disponibilidad y la capacidad de fijación de P del suelo (Fixen, 2005). La intensificación de la agricultura así como el uso de altas dosis de $\mathrm{P}$ ha permitido, a través de los años, incrementar los niveles de este nutriente, lo cual permitiría su aplicación al voleo e incorporado y reducir la dosis de $\mathrm{P}$ en las siembras de trigo.

Hay que considerar que los distintos genotipos de trigo difieren significativamente en su contenido de fósforo en las diferentes estructuras de la planta y de acuerdo al estado de desarrollo en que se encuentren (Barriga et al., 1990; Clarke et al., 1990; Mihaljev y Kastori, 1983; Romer y Schilling, 1986; Sutton et al., 1983).

Blair (1993); Mellado et al., (1991) y Batten (1992) describen distintos parámetros de eficiencia de asimilación del fósforo en trigo, tales como el índice de cosecha de fósforo (PHI) y la relación de eficiencia de fósforo $(\mathrm{PE}=$ rendimiento por unidad de P absorbido); sin embargo, no estudiaron el comportamiento de la planta en suelos con diferentes disponibilidades del elemento.

Por otra parte, la eficiencia del fósforo del trigo ha sido medida bajo un solo nivel de fertilización; pocos esfuerzos se han realizado para expresar la eficiencia en términos que incluyan varias dosis de fertilización fosfatada, lo cual puede ser más útil y apropiado para las condiciones de campo (Barriga, 1998).

En consideración a lo señalado, el objetivo de la presente investigación fue determinar el efecto de la forma de aplicación de mezclas con distintos contenidos de $\mathrm{P}$, en un suelo andisol con diferente disponibilidad inicial de P Olsen.

\section{MATERIALES Y MÉTODOS}

El experimento se realizó durante tres temporadas, 2005, 2006 y 2007, en un suelo andisol de la serie Freire, IX Región, descrito por Mella y Kuhne (1985), en el área agroclimática del llano central descrita por Rouanet (1983) como mediterráneo frío. Sus principales características químicas se indican en la Tabla 1.

Tabla 1

Características químicas del suelo utilizado

\begin{tabular}{|l|c|c|c|}
\hline \multicolumn{1}{|c|}{ Análisis } & $\mathbf{2 0 0 5}$ & $\mathbf{2 0 0 6}$ & $\mathbf{2 0 0 7}$ \\
\hline pH en agua & 5,93 & 5,87 & 5,89 \\
\hline M. orgánica $(\%)$ & 15 & 16 & 15 \\
\hline P Olsen $(\mathrm{mg} / \mathrm{kg})$ & 24 & 20 & 14 \\
\hline $\mathrm{K}(\mathrm{mg} / \mathrm{kg})$ & 203 & 176 & 78 \\
\hline $\mathrm{Ca}(\mathrm{cmol} / \mathrm{kg})$ & 6,05 & 5,76 & 5,87 \\
\hline $\mathrm{Mg}(\mathrm{cmol} / \mathrm{kg})$ & 0,99 & 0,90 & 0,83 \\
\hline Sat de Al $(\%)$ & 0,76 & 0,54 & 0,96 \\
\hline $\mathrm{S}(\mathrm{mg} / \mathrm{kg})$ & 10 & 11 & 11 \\
\hline B (mg/kg) & 0,72 & 0,50 & 0,61 \\
\hline Al extractable $\mathrm{mg} / \mathrm{kg}$ & 578 & 621 & 656 \\
\hline
\end{tabular}

El ensayo tuvo seis tratamientos, incluidos los testigos y cuatro repeticiones (Tabla 2).

Tabla 2

Tratamientos propuestos en los ensayos de campo

\begin{tabular}{|l|r|}
\hline \multicolumn{1}{|c|}{ Tratamientos } & Subtratamientos \\
\hline & $0 \mathrm{~kg} \mathrm{P}_{2} \mathrm{O}_{5}$ \\
Mezcla al surco de siembra & $70 \mathrm{~kg} \mathrm{P}_{2} \mathrm{O}_{5}$ \\
Mezcla al voleo e incorporada & $140 \mathrm{~kg} \mathrm{P}_{2} \mathrm{O}_{5}$ \\
& \\
\hline
\end{tabular}


El diseño experimental fue parcelas divididas en bloques distribuidos completamente al azar con un arreglo factorial $2 \times 3$, en parcelas de $12 \mathrm{~m}^{2}$ ( 2 x 6). Se utilizó la variedad Dollinco INIA de hábito de desarrollo alternativo. Se fertilizó a la siembra con $26 \mathrm{~kg} \mathrm{~N} / \mathrm{ha} ; 70 \mathrm{~kg} \mathrm{~K} 2 \mathrm{O} / \mathrm{ha} ; 18 \mathrm{~kg}$ $\mathrm{MgO} / \mathrm{ha} ; 22 \mathrm{~kg} \mathrm{~S} / \mathrm{ha}$ y $1,0 \mathrm{~kg} \mathrm{~B} / \mathrm{ha}$. La mezcla se realizó con $162 \mathrm{~kg}$ de salitre sódico, $100 \mathrm{~kg}$ de sulpomag, $80 \mathrm{~kg}$ de cloruro de potasio y $10 \mathrm{~kg}$ de boronat 32. El P, según tratamiento, se aplicó con superfosfato triple.

Del total de la dosis de $\mathrm{N}$ aplicado a la siembra un cien por ciento fue nitrógeno nítrico. El resto del N hasta completar 193 unidades se realizó con $180 \mathrm{~kg}$ de urea al estado de Zadoks 21 y $335 \mathrm{~kg}$ de supernitro 25 a Zadoks 30.

Las variables medidas fueron rendimiento de grano y P Olsen a la cosecha, en base a una muestra compuesta $0-20 \mathrm{~cm}$ en el tratamiento al voleo e incorporado. Luego de verificar homogeneidad de varianza y distribución normal de los datos, se efectuó a un análisis de varianza utilizando SPSS (2004). Las medias se compararon con la prueba de de Tuckey $(\mathrm{p} \leq 0,05)$.

\section{RESULTADOS Y DISCUSIÓN}

Rendimiento del grano: Al comparar los promedios de la forma de aplicación del fósforo, los resultados no presentaron diferencias estadísticas $(p>0,05)$ en los tres años evaluados (Figura 1).

Los resultados concuerdan con los trabajos publicados por Berardo (1999); Baumer et al. (2000) y Rillo (2006), quienes encontraron que en suelos con contenidos moderadamente altos de $\mathrm{P}$ las aplicaciones al voleo incorporado tuvieron el mismo rendimiento que las realizadas al surco. Por otro lado, Rodríguez et al. (2001) sostienen que las ventajas de las aplicaciones localizadas de $\mathrm{P}$ sobre las aplicaciones al voleo tienden a decrecer a medida que se incrementa el contenido de P Olsen en el suelo.

Respecto a las dosis de $\mathrm{P}$ utilizadas no se produjeron, en las temporadas 2005 y 2006, diferencias significativas de rendimiento de grano entre las diversas dosis de P utilizadas; sin embargo, en 2007 sí se observaron respuestas significativas a las dosis de $\mathrm{P}$, al aplicar 70 y $140 \mathrm{~kg}$ de $\mathrm{P} / \mathrm{ha}$ con respecto al testigo (Figura 2).
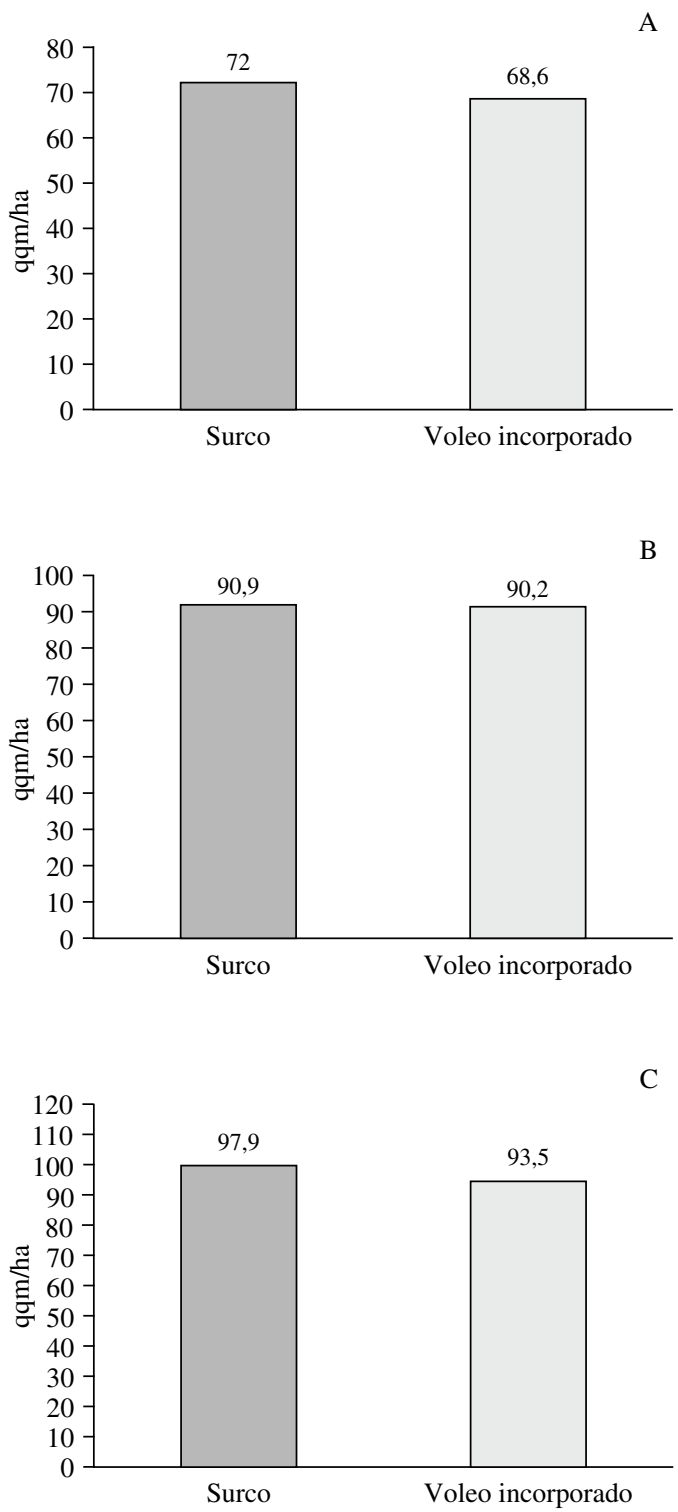

Figura 1. Efecto promedio de la forma de aplicación de $\mathrm{P}$ en el rendimiento, en A: 2005 (24 mg/kg P), B: 2006 (20 mg/kg P) y C: 2007 (14 mg/kg P).

De acuerdo a lo señalado por Rodríguez et al. (2001), la disponibilidad de $\mathrm{P}$ para trigo se calcula en base al P Olsen y la eficiencia de absorción de $\mathrm{P}$ del cultivo, que para el caso del trigo es de 1,5 kilos de $\mathrm{P}$ por cada $\mathrm{mg}$ de $\mathrm{P}$ Olsen. Es decir, la disponibilidad de $\mathrm{P}$ para el cultivo en los años 2005 y 2006, de 24 y 20 $\mathrm{mg} / \mathrm{kg}$ de P Olsen respectivamente, fue de $36 \mathrm{y}$ 30 kilos de $\mathrm{P}$, cantidad suficiente para satisfacer 

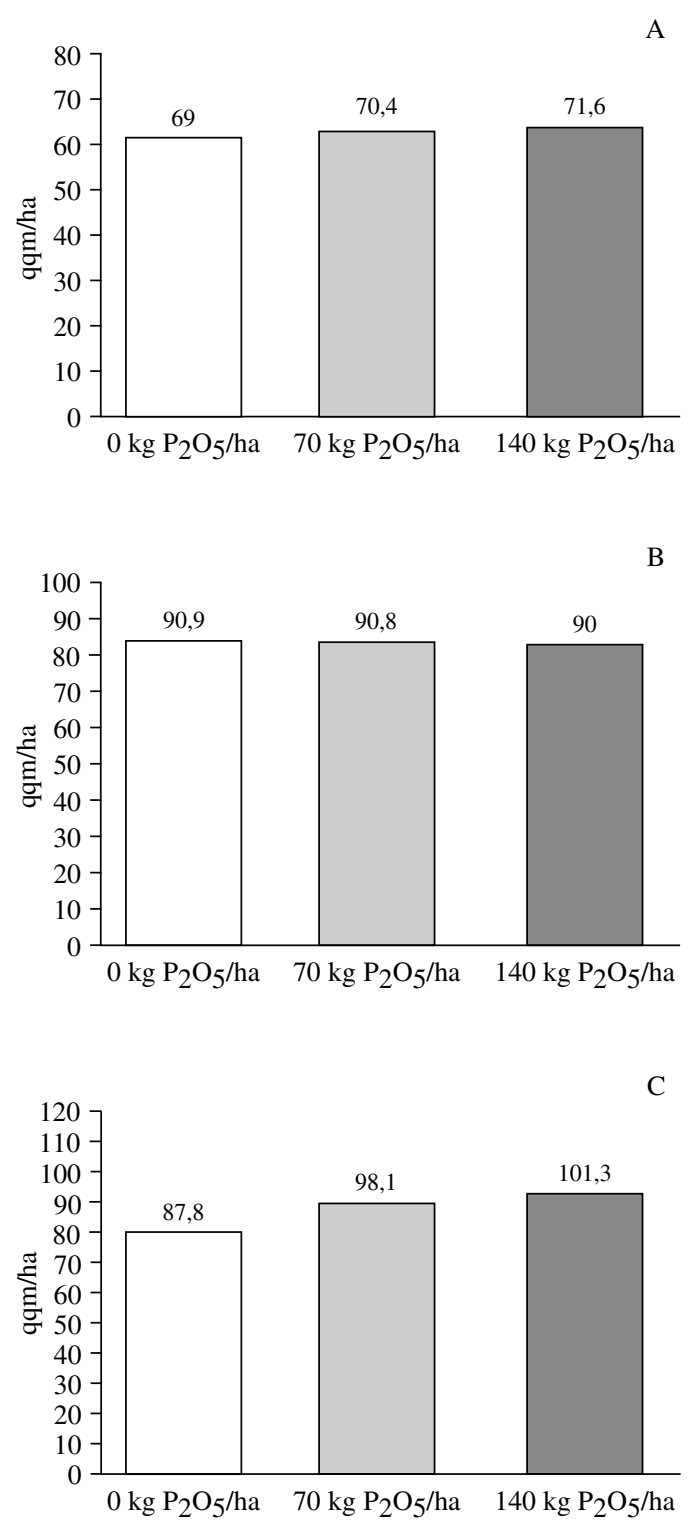

Figura 2. Efecto promedio de la dosis de $\mathrm{P}$ en el rendimiento, en A: 2005 (24 mg/kg P), B: $2006(20 \mathrm{mg} / \mathrm{kg} \mathrm{P})$ y C: 2007 $(14 \mathrm{mg} / \mathrm{kg}$ P).

los requerimientos de 23 kilos de $\mathrm{P}$, el año 2005 y de 29 kilos para el 2006, teniendo como base un requerimiento de 0,32 kilos de $\mathrm{P}$ por cada 100 kilos de trigo.

La disponibilidad de $\mathrm{P}$ el año 2006 de 14 $\mathrm{mg} / \mathrm{kg}$, equivalente a 21 kilos de $\mathrm{P}$ absorbibles para el trigo, fue insuficiente para satisfacer el requerimiento promedio de $32 \mathrm{~kg}$ de $\mathrm{P}$ del cultivo, motivo por el cual se produjo una respuesta significativa de rendimiento a favor de $70 \mathrm{y}$ $140 \mathrm{~kg}$ de $\mathrm{P}_{2} \mathrm{O}_{5} / \mathrm{ha}$.

Concordando con los resultados obtenidos, Bianchini (2003) y Bordoli et al. (2004) afirman que las aplicaciones de fertilizantes fosfatados solubles aplicados al voleo e incorporados antes de la siembra tuvieron respuestas similares a las de las aplicaciones en bandas al momento de la siembra, aun en suelos de baja disponibilidad de $\mathrm{P}$ extractable. Estas aplicaciones al voleo e incorporadas presentan ventajas desde el punto de vista práctico ya que incrementan la capacidad de trabajo de los equipos y podrían reducir la variabilidad en la distribución horizontal del P y, por lo tanto, los problemas asociados al muestreo y diagnóstico de la fertilidad.

P Olsen: En la temporada 2005 no se observó una tendencia clara en los contenidos de P Olsen, por efecto de las diferentes dosis de $\mathrm{P}$ utilizadas, $\mathrm{y}$ en las temporadas 2006 y 2007 se produjo un claro incremento del P Olsen (Figura 3).

De acuerdo a estudios citados por Rodríguez et al. (2001), para un suelo trumao en un rango de 400 a $800 \mathrm{mg} / \mathrm{kg}$ de aluminio extractable se esperaría un aumento de $1 \mathrm{mg} / \mathrm{kg}$ de P Olsen por cada $52 \mathrm{~kg}$ de $\mathrm{P}_{2} \mathrm{O}_{5}$ aplicados en un suelo trumao, sin considerar la extracción del cultivo. Al considerar el valor promedio de las tres temporadas el incremento con $70 \mathrm{~kg}$ de $\mathrm{P}_{2} \mathrm{O}_{5}$ fue de $2,0 \mathrm{mg} / \mathrm{kg}$ y de $2,3 \mathrm{mg} / \mathrm{kg}$ al aplicar $140 \mathrm{~kg}$ de $\mathrm{P}_{2} \mathrm{O}_{5}$, debiendo haber sido de 1,3 y $2,6 \mathrm{mg} / \mathrm{kg}$, respectivamente. Probablemente esta situación esté relacionada con la alta variabilidad espacial que presenta el P Olsen en los suelos.

\section{CONCLUSIONES}

No se produjeron diferencias significativas en rendimiento de trigo por efecto de la diferente forma de aplicación de la mezcla fertilizante, y tampoco por efecto de las diferentes dosis de $\mathrm{P}$ contenidas en las mezclas en suelos con 20 y $24 \mathrm{mg} / \mathrm{kg}$ de $\mathrm{P}$ Olsen. Sí se presentaron diferencias significativas de rendimiento cuando la disponibilidad de $\mathrm{P}$ fue de $14 \mathrm{mg} / \mathrm{kg}$.

Los resultados permitirían sugerir que en suelos con valores de $20 \mathrm{mg} / \mathrm{kg}$ de $P$ y superiores es factible aplicar las mezclas al voleo e incorporadas, sin que se registren diferencias de rendimiento en comparación a las aplicaciones al surco de 


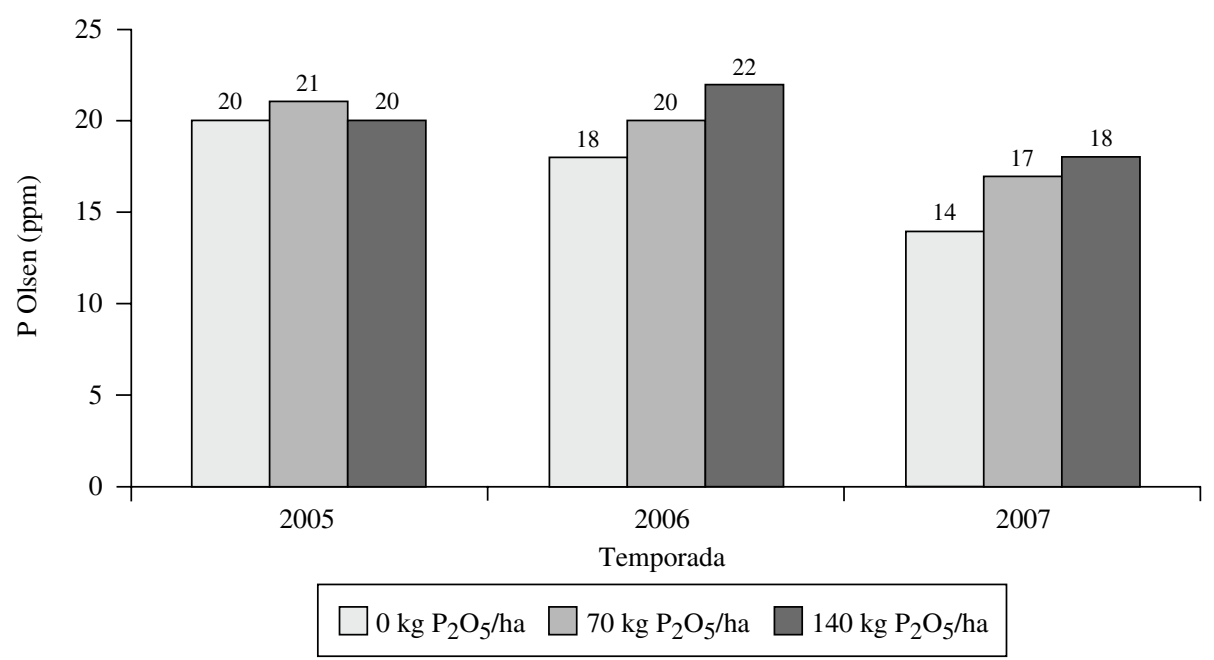

Figura 3. Efecto de las diferentes dosis de $\mathrm{P}$ aplicadas al voleo sobre la disponibilidad de $\mathrm{P}$ Olsen a la cosecha.

siembra. Además se podría reducir la dosis de $\mathrm{P}$ sin que se afecte el rendimiento.

El nivel de P Olsen del suelo, en general, se incrementó a medida que aumentó la dosis de $\mathrm{P}$ en la mezcla de siembra aplicada al voleo e incorporada.

\section{LITERATURA CITADA}

BARRIGA, P.; FUENTES, R.; SEEMANN, P.; MANQUIAN, N. 1990. Variabilidad genética de mulantes de trigo en la absorción y utilización de fósforo. Turrialba 40 : 279-286.

BARRIGA, P. 1998. Capacidad de absorción y utilización del fósforo en genotipos de trigo. Agro sur, 1998, Vol. 26, N 1 , pp. 1-10. ISSN 0304-8802.

BATTEN, G. 1992. A review of phosphorus efficiency in wheat, Plant and Soil 146:163-168.

BAUMER, C.; GONZÁLEZ, N.; DEVITO, C.; GIUFFRE, L.; RATTO, S.; PASCALE, C. 2000. Distribución del $\mathrm{P}$ extractable y respuesta del trigo en siembra directa a la fertilización localizada y al voleo. Rev. Tec. Agropecuaria. V (13): 9-11. EEA INTA Pergamino, Buenos Aires, Argentina.

BERARDO, A. 1999. Fertilización fosfatada de trigo: Respuesta y forma de aplicación. Informaciones Agronómicas, Instituto de la Potasa y el Fósforo. (2): 1-3, 1999.

BIANCHINI, A. 2003. Localización de fósforo en siembra directa. Actas Simposio "El fósforo en la agricultura argentina". INPOFOS Cono Sur. Acassuso, Buenos Aires, Argentina, pp. 79-82.

BLAIR, G. 1993. Nutrient efficiency - what do we really mean. In: Randall, P.J., Delhaize, E., Richards, R.A. y Munns, R. (eds.). Developments in Plant and Soil Sciences. Vol. 50 .
Para un manejo exitoso de aplicaciones de mezclas fertilizantes al voleo e incorporadas se requiere de un proceso de muestreo de suelo que garantice la calidad de la muestra y que sus resultados concuerden con el historial de manejo del suelo.
BORDOLI, J.M.; QUINCKE, A.; MARCHESSI, A. 2004. Fertilización fosfatada de trigo en siembra directa. XIX Congreso Argentino de la Ciencia del Suelo. Actas CD. AACS, Paraná, Entre Ríos, Argentina.

Clarke, J.; CAMPBElL, C.; CUTFORTH, H. 1990. Nitrogen and phosphorus uptake, translocation, and utilization efficiency of wheat in relation to environment and cultivar yield and protein levels. Can. J. Plant Sci. 70: 965-977.

DARWICH, N. 1998. Fósforo, un nutriente esencial para las plantas. En: Manual de fertilidad de suelos y uso de fertilizantes, pp. 65-94.

FARIÑA, J. 1997. Soja, la importancia de la ubicación del fertilizante. Revista Fertilizar No 8, pp. 4-7.

FIXEN, P. 2005. The application ways of phosphorus on the ground. Potash \& Phosphate Institute (PPI). (Available on www.infoagro.com/abonos/fosforo_suelo.htm).

MALLARINO, A. 1997. Manejo de fósforo, potasio y starters para maíz y soja en siembra directa. $5^{\circ}$ Congreso Nacional de AAPRESID. Mar del Plata, pp. 11-19.

MALLARINO, A. 1998. Métodos de fertilización con fósforo y potasio para maíz y soja en siembra directa: Recientes avances en el cinturón del maíz. $6^{\circ}$ Congreso Nacional de AAPRESID, pp. 27-41.

MELLA, A.; KUHNE, G. 1985. Sistema de descripción de las familias, asociaciones y series de suelos derivados 
de materiales piroclásticos de la zona central-sur de Chile. En: J. Tosso (Ed.). Suelos Volcánicos de Chile. INIA. Ministerio de Agricultura. Santiago. Chile. Cap. 1, pp. $25-95$.

MALLARINO, A.; BORGES, R. 2006. Phosphorus and potassium distribution in soil following long-term deepband fertilization in different tillage systems. Soil Sci. Soc. Am. J. 70:702-707.

MELLADO, M.; RODRÍGUEZ, N.; GUTIÉRREZ, G. 1991. Selección preliminar de variedades de trigo (Triticum aestivum L.) sobre la base de su capacidad de absorber el fósforo nativo y aplicado. Agricultura Técnica 51: 280-282.

MIHALJEV, I.; KASTORI, R. 1983. Genotypic variation and inheritance of mineral element content in winter wheat. In: Saric, M.R. y Loughman, B.C. (eds.). Genetic Aspects of Plant Nutrition. Marlinus Nijhoff. The Hague, pp. 477-481.

RILLO, S. 2006. Evaluación de dos formas de aplicación del fósforo en suelos Hapludoles énticos en siembra directa. Resultados de experiencias campaña 2005-2006. INTA publicaciones regionales.
RODRÍGUEZ, J.; PINOCHET, D.; MATUS, F. 2001. La fertilización de los cultivos. Impreso en LOM Ediciones, Santiago de Chile, 2001.

ROMER, W.;SCHILLING, G. 1986. Phosphorus requeriments of the wheat plant in various stage of its life cicle. Plant and Soil 91: 221-229.

ROUANET, J. 1983. Clasificación agroclimática de la IX Región. Segunda aproximación. Investigación y progreso Agropecuario. Carillanca 2 (4): 25-27.

SUTTON, P.; PETERSON, G.; SANDER, D. 1983. Dry matter production in tops and roots of winter wheat as affected by phosphorus availability during various growth stages. Agron. J. 75: 657-663.

SPSS. 2004. Statistical Package for the Social Sciences (SPSS). SPSS Base 12.0 User's Guide for Windows. SPSS Inc., Chicago, Illinois, USA. Disponible en http://www.spss. $\mathrm{com} / / \mathrm{spss}$

ZADOKS, J.; CHANG, T.; KONZAK, C. 1974. Código decimal para los estados de crecimiento de cereales. Weed Research. 14: 415-421. 